\title{
Functional interaction between FOXO3a and ATM regulates DNA damage response
}

\author{
Wen-Bin Tsai ${ }^{1}$, Young Min Chung ${ }^{1}$, Yoko Takahashi ${ }^{1}$, Zhaohui $\mathrm{Xu}^{1}$, and Mickey C-T. Hu ${ }^{1,2,3}$ \\ 1Departments of Molecular and Cellular Oncology, The University of Texas M. D. Anderson Cancer \\ Center, Houston, Texas 77030, USA
}

2 Graduate School of Biomedical Sciences, The University of Texas M. D. Anderson Cancer Center, Houston, Texas 77030, USA

\begin{abstract}
The maintenance of genomic stability in cells is relentlessly challenged by environmental stresses that induce DNA breaks, which activate the DNA-damage pathway mediated by protein complexes such as ataxia-telangiectasia mutated (ATM) and its downstream mediators to sense DNA damage and control damage-induced cell-cycle checkpoints and DNA repair ${ }^{1-3}$. Here we show that FOXO3a interacts with ATM to promote phosphorylation of ATM at Serine-1981 (ATM-pS1981) and to prompt its downstream mediators to form nuclear foci in response to DNA damage. Silencing FOXO3a in cells abrogates the formation of ATM-pS1981 and phospho-histone H2AX foci after DNA damage. Increasing FOXO3a in cells promotes ATM-regulated signaling, intra-S-phase or $\mathrm{G}_{2} / \mathrm{M}$ cell-cycle checkpoint, and repair of damaged DNA, whereas cells lacking FOXO3a could not trigger the DNA-repair mechanism after DNA damage. Interestingly, the carboxy-terminal domain of FOXO3a binds to the FAT domain of ATM, thereby contributing to the activation of ATM. These results suggest that ATM may be regulated directly by FOXO3a in DNA damage response.
\end{abstract}

DNA damage occurs frequently and accumulates with age in cells, and the genetic factors controlling DNA repair induced by environmental stresses regulate the aging process, ageassociated diseases, cancer development, and longevity in mammals. A key factor, ataxiatelangiectasia mutated (ATM), is activated upon DNA damage ${ }^{4-6}$ and that activation of ATM induces phosphorylation of its downstream target histone-H2A variant $\mathrm{H} 2 \mathrm{AX}(\gamma-\mathrm{H} 2 \mathrm{AX})$, leading to the recruitment of DNA repair proteins to the sites of DNA damage ${ }^{7,8}$. Although an important ATM signaling pathway through the Mre11-Rad50-Nbs1 (MRN) complex is known 6,9 , further elucidation of the mechanism underlying the control of ATM activation is necessary for understanding the ATM-mediated DNA damage response.

The mammalian forkhead box-O (FOXO) transcription factors, homologues of DAF-16 that regulates longevity in Caenorhabditis elegans, are factors that play a key role in regulating cell-cycle and death control ${ }^{10-13}$. FOXO factors are negatively regulated by the survival signaling pathways through $\mathrm{Akt}^{10-13}$ or IkB-kinase (IKK) ${ }^{13,14}$ or other kinases ${ }^{10-13}$, resulting in advancement through the cell cycle. Upon DNA damage, cells trigger cell-cycle

3Correspondence should be addressed to M.C.T.H. (e-mail: E-mail: michu@mdanderson.org).

Authors' contributions

WBT carried out the majority of experiments including the cell-cycle checkpoint, siRNA knockdown, co-immunoprecipitation, immunoblotting, immunofluorescence confocal microscopy, tumour staining, GST pull-down, immunocomplex kinase assays, and data analysis. YMC performed all the analysis of DNA damage by the comet assays and quantification of immunofluorescence images. YT conducted quantitative real-time PCR analyses, mammalian and yeast two-hybrid assays. ZX provided crucial assistance in generating DNA constructs, cell lines, GST fusion proteins, and performing luciferase and PCR analyses. MCTH generated DNA constructs and cell lines, designed and coordinated all experimental approaches, drafted and revised the entire manuscript. 
checkpoints at $\mathrm{G}_{1} / \mathrm{S}$ - or $\mathrm{G}_{2} / \mathrm{M}$-phase to prevent cell-cycle progression, which provides the cellular DNA-repair machinery enough time to repair the damaged DNA. Because defects in the proper regulation of cell-cycle checkpoints may lead to the accumulation of DNA lesions that promote cancer development, it is fundamentally important to identify key genetic factors that control cell-cycle checkpoints and sustain the integrity of the genome in cells. Overexpression of FOXO in cells inhibits cell-cycle progression and protects cells against oxidative damage or genotoxic stress ${ }^{10,15-17}$. However, the role of endogenous FOXO in controlling complex cell-cycle checkpoints after initial DNA double-strand breaks remains unclear.

To examine the role of endogenous FOXO3a in cell-cycle progression after DNA damage, we generated MCF-7 and LNCaP FOXO3a-knockdown (MCF7-Fd and LNCaP-Fd) and FOXO3a-overexpression (MCF7-FO and LNCaP-FO) stable cell lines (SupplementaryInformation, Fig. S1a,b). We showed that ionizing radiation (IR) induced decreases in the incorporation of bromodeoxyuridine (BrdU) (i.e., S-phase arrest) in both MCF-7 and LNCaP cells in a dose-dependent manner. However, this IR-induced S-phase arrest ${ }^{18}$ was abrogated in MCF7-Fd-Pc and LNCaP-Fd cells (Fig. 1a,b; Supplementary-Fig. S1c,d), suggesting that IR induces an S-phase arrest and that silencing FOXO3a results in a defective IR-induced intra-S-phase checkpoint.

To assess the $\mathrm{G}_{2} / \mathrm{M}$-checkpoint, we treated MCF-7 and various MCF7-Fd cell lines with either IR or camptothecin (CPT) to induce DNA damage. Although CPT or IR reduced the entry of MCF-7 into M-phase, CPT or IR enhanced the entry of MCF7-Fd cells into M-phase (Fig. $1 \mathrm{c}, \mathrm{d})$. These results were confirmed in three independent single clones of MCF7-Fd and MCF7FO33 (Supplementary-Fig. S1e-h). To generalize the observed effects, we did the same experiments with another cell line, $\mathrm{LNCaP}$, and showed that CPT and IR decreased the entry of LNCaP into M-phase, whereas CPT and IR increased the entry of LNCaP-Fd cells into Mphase (Fig. 1e,f; Supplementary-Fig. S1i,j), suggesting that silencing FOXO3a results in a deficient CPT- or IR-induced $\mathrm{G}_{2} / \mathrm{M}$-checkpoint.

To examine the effect of FOXO3a on activation of the ATM-mediated DNA damage response pathway, we compared the level of phosphorylation of ATM at Serine-1981 (ATM-pS1981) among MCF-7, MCF7-Fd, and MCF7-FO cell lines treated with CPT or DMSO by flow cytometry. The ATM-pS1981-positive cell populations increased in MCF-7 and MCF7-FO33 treated with CPT; however, no such increase was observed in MCF7-Fd-Pa and MCF7-Fd-Pc (pooled clones) treated with CPT (Supplementary-Fig. S1k). Similarly, the proportion of $\gamma-$ H2AX-positive cells paralleled the level of FOXO3a expression in those cells (SupplementaryFig. S11), suggesting that FOXO3a may have a role in activation of the ATM-response pathway after DNA damage. The results obtained by flow cytometry were confirmed by immunoblotting analysis of MCF-7 transfected with siRNA targeting FOXO3a and of MCF7-Fd-Pc cell line after IR (5-Gy) for 5min or 10min (Supplementary-Fig. S1m,n).

To determine whether FOXO3a is involved in ATM activation at the very early stages of response to DNA damage, we treated MCF-7 cells briefly with IR (5- or 3-Gy) or CPT and analyzed the levels of $\gamma-\mathrm{H} 2 \mathrm{AX}$ and ATM-pS1981 and phosphorylation of its downstream $\mathrm{G}_{2} /$ $\mathrm{M}$-targets such as BRCA1 and hRad17 2,19 in the nuclear and cytoplasmic extracts. We found that phosphorylation of S1981-ATM could be induced early (5min) after IR (5- or 3-Gy) or CPT treatment in MCF-7 cells (Supplementary-Fig. S2a-c). We also performed the same experiments with another cell line, LNCaP, and confirmed that phosphorylation of S1981ATM could be induced shortly after a physiologically relevant dose (5-Gy) of radiation (i.e., 5min after IR) (Supplementary-Fig. S2d). In addition, we showed that phosphorylation of BRCA1 (BRCA1-pS1423) and hRad17 (hRad17-pS645) was induced in parallel with phosphorylation of S1981-ATM in MCF-7 cells shortly after DNA damage (5min after IR or CPT) (Supplementary-Fig. S2a-c) and in LNCaP cells after IR (5-Gy) (Supplementary-Fig. 
S2d). These increases in BRCA1-pS1423 and hRad17-pS645 after DNA damage were abrogated in the FOXO3a-knockdown MCF-7 cells (Supplementary-Fig. S1m), suggesting that FOXO3a is necessary for promoting the phosphorylation of these molecules at these sites in response to DNA damage.

Since it has been shown that ATM can bind to NEMO in the nucleus after DNA damage and hence activate IKK signaling ${ }^{20}$, we examined the levels of NEMO and IKK in the cytoplasmic and nuclear extracts of LNCaP and MCF-7 cells after DNA damage by IB analysis. We showed that the levels of NEMO and NEMO phosphorylated at serine-85 (NEMO-pS85) did not change in the cytoplasm and nucleus of LNCaP after IR at 10-Gy or MCF-7 after CPT, but the levels of ATM phosphorylated at S1981 were greatly elevated in the nuclei of LNCaP cells at $5 \mathrm{~min}$ after irradiation (Supplementary-Fig. S2e). Similarly, the levels of NEMO and NEMO-pS85 did not change in the cytoplasm and nucleus of MCF-7 after CPT, but the levels of ATMpS1981 were increased in the nuclei of MCF-7 after CPT (Supplementary-Fig. S2f). As a CPT control, DMSO did not alter the levels of the tested proteins in cell lysates (SupplementaryFig. S2g). We further showed that the levels of IKK $\alpha / \mathrm{IKK} \beta$ did not change in the cytoplasm or the nucleus of LNCaP after IR (10-Gy) or MCF-7 after CPT (Supplementary-Fig. S2e,f), indicating that neither NEMO nor IKK is involved in the FOXO3a-mediated activation of the ATM DNA-damage response.

Because $\gamma-\mathrm{H} 2 \mathrm{AX}$ is associated with chromatin domains flanking the sites of DNA doublestrand breaks and plays a key role in recruiting DNA repair proteins to nuclear foci containing the sites of damage ${ }^{6-8}$, we assessed whether endogenous FOXO3a could localize to sites of DNA breaks by co-localization with $\gamma$-H2AX. MCF-7 cells were treated with IR (5-Gy for $10 \mathrm{~min}$ ) and stained with antibodies against FOXO3a and $\gamma$-H2AX. We found that $\sim 80 \%$ of FOXO3a could co-localize with $\gamma$-H2AX foci in the nucleus after exposure to IR, whereas more than $90 \%$ of FOXO3a remained in the cytoplasm of cells untreated (Supplementary-Fig. S3a). Similarly, 76\% of FOXO3a could co-localize with ATM-pS1981 foci in the nucleus after exposure to IR (5Gy for 10min) (Supplementary-Fig. S3b), suggesting that FOXO3a may localize to sites of DNA breaks in response to DNA damage. Since $\gamma$-H2AX/ATM-pS1981 nuclear foci could co-localize with FOXO3a after DNA damage (Fig. 2a,b; SupplementaryFig. S3a-d), we examined the ability of these phospho-proteins to form nuclear foci in the absence of FOXO3a. Strikingly, the formation of $\gamma$-H2AX and ATM-pS1981 nuclear foci was abrogated in MCF-7 in which FOXO3a was silenced by siRNA after exposure to CPT for 10min, whereas many nuclear foci of $\gamma-\mathrm{H} 2 \mathrm{AX}$ and ATM-pS1981 could form in the presence of FOXO3a in wild-type cells after treatment (Fig. 2c,d; Supplementary-Fig. S3e-h).

To verify that FOXO3a is indeed present in nuclear DNA damage foci but does not reflect its being trapped in the nucleus independent of damage ${ }^{21}$, we compared subcellular localization of FOXO3a in the presence of LY294002 (an inhibitor of phosphatidylinositol-3-kinase (PI3K)-Akt signaling) with that in the absence of LY294002 in cells without DNA damage. Our results showed that in the absence of IR but with LY294002, FOXO3a was located primarily in the nucleus (Supplementary-Fig. S3i,k), whereas without LY294002 most of the FOXO3a was in the cytoplasm (Supplementary-Fig. S3a-d). Cells treated with LY294002 without IR did not show staining for $\gamma$-H2AX or ATM-pS1981 (Supplementary Fig. S3i-1), suggesting that LY294002 can induce FOXO3a nuclear localization but cannot activate $\gamma$ H2AX and ATM-pS1981. In the presence of both IR (5Gy) for 10min and LY294002, $\gamma$-H2AX and ATM-pS1981 were activated and could co-localize with FOXO3a in the nucleus (Supplementary Fig. S3i-1). These findings are consistent with the results without LY294002 (Supplementary-Fig. S3a-d) and confirm that the observed IR-induced co-localization of FOXO3a with $\gamma$-H2AX/ATM-pS1981 is independent of the PI3K-Akt pathway, suggesting that FOXO3a is indeed present in nuclear DNA damage foci. Moreover, co-localization of FOXO3a with ATM-pS1981 or $\gamma-\mathrm{H} 2 \mathrm{AX}$ was also observed in human primary tumour tissues 
in vivo (Fig. 3a,b; Supplementary-Fig. S3m,n), suggesting that FOXO3a may be important in the recruitment of key ATM-signaling mediators to sites of DNA damage and that this mechanism may be physiologically or pathologically significant.

To confirm the protein interactions between FOXO3a and ATM-pS1981or its downstream $\mathrm{G}_{2} / \mathrm{M}$-targets, we treated MCF-7 with CPT and performed co-immunoprecipitation with antibodies to FOXO3a or ATM-pS1981 or $\gamma$-H2AX followed by IB analysis. Endogenous FOXO3a was specifically associated with ATM-pS1981, $\gamma$-H2AX, BRCA1-pS1423, and hRad17-pS645 after CPT treatment in a time-dependent manner (Fig. 3c-e; SupplementaryFig. S4a-d). To determine the ability of ATM-pS1981 to interact with $\gamma-\mathrm{H} 2 \mathrm{AX}$ in the absence of FOXO3a following DNA damage, we treated wild-type and FOXO3a(-l-) MEFs with CPT and performed the same co-IP experiments. The interaction between ATM-pS1981 and $\gamma$ $\mathrm{H} 2 \mathrm{AX}$ was abolished in FOXO3a(-/-) MEFs, but their interaction remained largely intact in wild-type MEFs (Supplementary-Fig. S4e,f), suggesting that FOXO3a may be necessary for the association between ATM-pS1981 and $\gamma$-H2AX upon DNA damage.

To examine the role of FOXO3a in regulating ATM-pS1981 through ATM

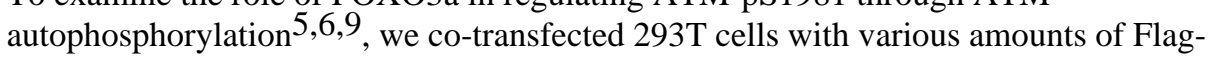
FOXO3a and control vectors and showed that the level of ATM-pS1981 was increased slightly in a FOXO3a-dependent manner without DNA damage. Similarly, the level of ATMpS1981was enhanced in MCF7-FO33 and MCF7-FO41 cells relative to control cells (Supplementary-Fig. S4g,h). The level of ATM-pS1981or $\gamma$-H2AX was increased after the addition of FOXO3a protein into an ATM protein complex in vitro in a dose-dependent fashion (Fig. 3f). Although the level of ATM-pS1981 was enhanced by the addition of linear doublestranded DNA fragments (mimicking double-strand breaks), the increase of ATM-pS1981 was abrogated in the presence of protein phosphatase (Supplementary-Fig. S4i). This confirms that the observed increase of ATM-pS1981 took place through protein phosphorylation. To further validate the direct role of FOXO3a protein in regulating the level of ATM-pS1981 in vitro, we added purified recombinant glutathione-S-transferase (GST) -FOXO3a protein that was free of DNA into the same ATM protein complex and showed that the level of ATM-pS1981 was enhanced by GST-FOXO3a protein in a dose-dependent manner but not by GST (negative control) (Supplementary-Fig. S4j), suggesting that FOXO3a protein may promote ATM autophosphorylation.

Because activation of ATM-pS1981 and the intra-S-phase or $\mathrm{G}_{2} / \mathrm{M}$-checkpoint can trigger DNA repair, we next determined if FOXO3a could induce the repair of damaged DNA. We used a standard comet assay to measure the percentage of damaged DNA templates that had been repaired. We found that overexpression of FOXO3a in MCF-7 cells led to augmented repair of damaged DNA (i.e., reduced the comet tail moment) after CPT or IR, whereas knocking out FOXO3a in MEFs significantly inhibited the repair of damaged DNA (i.e., increased the comet tail moment) upon CPT or IR (Fig. 4a-d). We also measured the percentage of damaged DNA templates that had been repaired by using real-time quantitative polymerase chain reaction (PCR)-based assay ${ }^{23}$ or standard PCR amplification in the linear range. We cotransfected the indicated cells with a pre-damaged DNA template pGL2-B and an undamaged control circular DNA template pGEX. The percentage of repair of the damaged template pGL2$\mathrm{B}$ was determined by the ratio of the real-time quantitative PCR amplification products with Luc-specific primers on the repaired Luc template versus those with pGEX-specific primers on the undamaged template as PCR controls. The relative level of repair of the damaged template was significantly enhanced in MCF7-FO33 cells relative to that in MCF7-C5 cells (Supplementary-Fig. S5a-d), whereas DNA repair was repressed in FOXO3a(-/-) MEFs relative to wt MEFs (Supplementary-Fig. S5e-h). 
To elucidate the possible mechanism by which FOXO3a might promote ATM autophosphorylation and activation, we examined the binding between FOXO3a and ATM by using standard GST pull-down assays. The results showed that the conserved cysteine (C) and aspartic acid (D) residues within the carboxy-terminal domain (amino acids[a.a.]616-623) of FOXO3a bind to ATM (Fig. 5a-e; Supplementary-Fig. S6a,b). Mutation of either or both residues in this domain abrogated the binding of FOXO3a to ATM, suggesting that this "CD" motif is necessary for the interaction of FOXO3a and ATM. In addition, we found that FOXO3a binds ATM at a region (a.a.1764-2841) that covers a putative "FAT" domain (a.a.1960-2566), which has been postulated as a protein-binding domain ${ }^{2,25}$ (Fig. 5f; Supplementary-Fig. S6c). Although this domain contains its autophosphorylation site Serine-1981, phosphorylation of this site did not seem to be involved in the interaction between ATM and FOXO3a. We further confirmed this binding in a mammalian two-hybrid system and narrowed the interaction between FOXO3a and ATM down to a smaller region (a.a.1802-2571) that mainly contains the "FAT" domain (Fig. 5g; Supplementary-Fig. S6c). In accordance with our GST-pull-down findings, mutation of either or both residues in this "CD" motif of FOXO3a suppressed the interaction between FOXO3a and $\operatorname{ATM}(1802-2571)$ in these assays (Fig. 5h). Collectively, these results suggest a new mechanism in which the $\mathrm{CD}$ motif within the carboxy-terminal domain of FOXO3a binds to the FAT domain of ATM, thereby contributing to the activation of ATM autophosphorylation.

Our findings suggest that ATM may be regulated directly by FOXO3a to promote repair of damaged DNA. ATM activation has been correlated with autophosphorylation of ATM at Serine-1981 in response to DNA damage ${ }^{5,6}$. Because our findings indicated that silencing FOXO3a prevents autophosphorylation and activation of ATM after DNA damage and leads to a deficient $\mathrm{G}_{2} / \mathrm{M}$-phase cell-cycle checkpoint, the implications of our results are consistent with the finding that ATM-knockout cells have a deficient $\mathrm{G}_{2} / \mathrm{M}$-checkpoint ${ }^{26}$. In terms of the response to DNA damage, DNA breaks could induce conformational changes in nuclear FOXO3a such that it detaches from the DNA and interacts with ATM in the nucleus soon after DNA damage. Subsequently, further DNA damage could trigger translocation of FOXO3a from the cytoplasm to the nucleus and augment the formation of more functional FOXO3a/ ATM protein complexes, leading to higher levels of activated ATM-pS1981, which in turn would phosphorylate its downstream mediators such as H2AX and execute DNA-repair programs. Although our results indicate that FOXO3a can promote the intra-S-phase or $\mathrm{G}_{2} / \mathrm{M}$ phase checkpoint to facilitate repair of damaged DNA in the presence of genotoxic stress, FOXO3a may have distinct roles in regulating cell-cycle checkpoints in response to DNAreplication stress occurring during cell proliferation 27 or DNA-damage stress induced by external genotoxic agents. In this respect, FOXO3a may promote the ATM-response network to facilitate DNA repair under short-term exposure to low levels of stress, whereas FOXO3a may trigger a death program ${ }^{12-15}$ under prolonged exposure to higher levels of stress. This orchestrated DNA repair program in response to environmental stresses would mediate not only timely DNA repair to prevent the most harmful lesions to the integrity of the genome but a death program to eliminate cells with heavily damaged DNA, leading to an increase in resistance to tumour development ${ }^{28}$ or the ageing process.

\section{METHODS}

\section{Antibodies and reagents}

The DNA damage agent CPT was purchased from Sigma (St. Louis, MO). The drug was dissolved in dimethylsulfoxide (DMSO) or ethanol and stored in aliquots at $-20^{\circ} \mathrm{C}$. Antibodies specific to FOXO3a (FKHRL1, H-144), FOXO1a (FKHR, H-128), FOXO4, IKK $\alpha / \beta$, IKK $\gamma$ (NEMO), PCNA, PTEN, and p53 (DO-1) were obtained from Santa Cruz Biotechnology (Santa Cruz, CA). Antibodies against phospho-ATM (Serine-1981), phospho-H2AX (Serine-139), 
phospho-BRCA1 (BRCA1-pS1423), phospho-Histone H3 (Serine-10), FOXO3a (FKHRL1, 06-951), NBS1, and Rad50 were purchased from Upstate Biotechnology (Lake Placid, NY). Antibodies against ATM (BL706 and BL116G), H2AX (BL179), BRCA1-pS1423, and BRCA1 were purchased from Bethyl Laboratories (Montgomery, TX). Antibodies against $\beta$ tubulin, $\beta$-actin, hemagglutinin (HA), and Flag were purchased from Sigma. An anti-p27Kip1 antibody was purchased from BD PharMingen (San Diego, CA). Antibodies against and phospho-hRad17 (hRad17-pS645) and hRad17 were purchased from Cell Signaling Technology (Danvers, MA). Antibodies against p53 (Ab-6) and poly-ADP-ribose polymerase were purchased from Oncogene (Boston, MA) and Roche Applied Science (Indianapolis, IN), respectively. A phospho-antibody against phospho-NEMO at serine-85 (NEMO-pS85) was kindly provided by S. Miyamoto (University of Wisconsin). Alexa 488 (green)- and Alexa 546 (red)-conjugated secondary antibodies were obtained from Molecular Probes (Eugene, OR).

\section{Cell culture and cell lines}

All cell lines were grown at $37^{\circ} \mathrm{C}$ and $5 \% \mathrm{CO}_{2}$ in DMEM/F12 supplemented with L-glutamine, penicilline/streptomycin and 10\% fetal bovine serum. MCF-7 stable cell lines with FOXO3a downregulated (MCF7-Fd) or overexpressed (MCF7-FO) and control cell lines (MCF7-Cv) were established with retroviruses. Specifically, retroviruses expressing short-hairpin (sh) RNA-FOXO3a were generated with a pre-made retroviral pSM2 construct (Open Biosystems) containing shRNA directed against human FOXO3a (V2HS_169297) according to the manufacturer's instructions. Retroviruses expressing HA-FOXO3a were made with pBabeHA-FOXO3a construct, which was created by ligating a BamHI-XhoI fragment of HAFOXO3a into a pBabe-puro vector cut with BamHI and SalI. MCF-7 cells were infected with retroviruses expressing shRNA-FOXO3a or expressing HA-FOXO3a or control vectors. LNCaP cells were infected with retroviruses expressing shRNA-FOXO3a or shRNA-Gadd45a or expressing HA-FOXO3a or control vectors. After puromycin selection $(0.15-0.5 \mu \mathrm{g} / \mathrm{ml})$, MCF-7 and LNCaP FOXO3a-knockdown pooled clones (MCF7-Fd-Pa, -Pc, and LNCaP-Fd) or individual clones (MCF7-Fd-1, Fd-25, Fd-35), MCF-7 and LNCaP FOXO3aoverexpressing clones (MCF7-FO33 or -FO41, and LNCaP-FO), and vector control clones (Cv-4, Cv-14, and C12) were selected. Wild-type (wt) and knockout MEF cells were kindly provided by R. Depinho, N. Motoyama, and A.J. Fornace Jr. as described in the Supplementary Materials and Methods.

\section{lonizing radiation and intra-S checkpoint assay}

Cells were seeded at $70 \%$ confluence $18-20 \mathrm{~h}$ before IR. Cells were g-irradiated in the presence of medium with a ${ }^{137} \mathrm{Cs}$ source emitting the indicated Gy at a fixed dose rate (Irradiator model 0103, U.S. Nuclear Corp.) in the absence of medium without the lid. After irradiation, the fresh medium was added and cells were incubated for the indicated times. For the intra-S checkpoint (S-phase arrest) assay, the analysis of DNA synthesis is based on the incorporation of BrdU into the genomic DNA. Cells were irradiated with $10 \mathrm{~Gy}$ of IR and incubated for $1 \mathrm{~h}$. Then cells were labeled with BrdU-labeling reagent $(10 \mu \mathrm{M})$ (Roche Applied Science) for 30min, harvested and fixed with ethanol, and then cellular DNA was denatured by $\mathrm{HCl}(4 \mathrm{~N})$ accordingto the manufacturer's instructions. After $\mathrm{pH}$ neutralization, cells were immunostained with anti-BrdU-FLUOS (Roche Applied Science) and $10 \mu \mathrm{g} / \mathrm{ml}$ propidium iodide (PI) (Roche Applied Science). The samples were analyzed by flow cytometry with a fluorescence-activated cell sorter (BD Bioscience, CA) and the Cell-Quest program.

\section{Cell cycle analysis}

Cells were treated with the DNA damage agent CPT $(1 \mu \mathrm{M})$ or control (DMSO) for $5 \mathrm{~h}$, or with IR at 0,5 , or $10 \mathrm{~Gy}$ for $1 \mathrm{~h}$. Cells were washed with phosphate-buffered saline (PBS) to remove CPT or DMSO and cultured for another $24 \mathrm{~h}$, allowing time for activating cell cycle 
checkpoints and repairing DNA. Cells were rinsed with PBS and fixed in $70 \%$ ethanol at $4{ }^{\circ} \mathrm{C}$ overnight. The fixed cells were then washed twice with PBS and resuspended in PBS containing $0.5 \%$ bovine serum albumin (BSA, Sigma). After centrifugation, the pellet was resuspended in $100 \mathrm{ml}$ of PBS containing 1\% BSA and a monoclonal antibody against phospho-ATM (Serine-1981) (1:200) or phospho-H2AX (Serine-139) (1:800) (Upstate) and incubated for 2 $\mathrm{h}$ at room temperature. After centrifugation, the cell pellets were washed with PBS and resuspended in $100 \mathrm{ml}$ PBS containing an anti-mouse or anti-rabbit Alexa 488 (green)conjugated secondary antibody (Molecular Probes) (1:400) for $1 \mathrm{~h}$ at room temperature in the dark. After washing, the cells were counterstained with $10 \mu \mathrm{g} / \mathrm{ml}$ PI (Roche Applied Science) containing $10 \mu \mathrm{g} / \mathrm{ml} \mathrm{RNase} \mathrm{A} \mathrm{(Sigma)} \mathrm{for} 1 \mathrm{~h}$ at room temperature before analysis. The samples were analyzed by flow cytometry as described above. Cells in mitosis were determined by staining with an antibody against phosphorylated histone H3 at Serine-10 (P-H3; Cell Signaling), which stains cells that are in the M phase, or an isotype $\operatorname{IgG}$ (negative control), followed by an Alexa 488-conjugated secondary antibody and PI. The percentage of the Mphase cells was determined by flow cytometry.

\section{Immunoprecipitation and immunoblotting}

All IP experiments were performed as described previously ${ }^{14}$. Briefly, cells were washed twice with PBS and lysed with lysis buffer containing protease inhibitors at $4^{\circ} \mathrm{C}$ for $20 \mathrm{~min}$. The lysates were centrifuged at $16,000 \mathrm{~g}$ for $10 \mathrm{~min}$ to remove cell debris. Total protein concentration was determined as described above. Protein samples were first precleared with a nonspecific $\mathrm{IgG}$ antibody. Precleared lysates were then incubated with an antibody by rotating at $4{ }^{\circ} \mathrm{C}$ overnight followed by the addition of $25 \mu \mathrm{l}$ of $50 \%$ protein A- or Protein G-sepharose slurry and rotating for $1 \mathrm{~h}$. Protein $\mathrm{A} / \mathrm{G}$ beads were collected and washed with lysis buffer four times. Immunoprecipitates were resolved by $6 \%$ or $10 \%$ or $12 \%$ SDS-polyacrylamide gel eletrophoresis (PAGE) and analyzed by IB analysis. Some immunoprecipitates were also subjected to high stringency washes in lysis buffer $(\mathrm{pH} 7.4)$ containing various concentrations $(0.15,0.3,0.5,0.7$, or $1 \mathrm{M})$ of $\mathrm{NaCl}$ in conjunction with various amounts $(0,0.1,0.5,1$, or $2 \%)$ of NP40. After washing, immunoprecipitates were resolved by SDS-PAGE and analyzed by IB analysis. For IB analysis, the protein samples were subjected to SDS-PAGE and transferred onto polyvinylidene difluoridemembranes. The membranes were blocked with $5 \%$ nonfat dry milk in PBS containing $0.05 \%$ Tween 20 and incubated with primary antibodies and then with horseradish peroxidase-conjugated secondary antibodies according to the manufacturer's instructions. The immunoblots were visualized by an enhanced chemiluminescence kit obtained from Amersham Pharmacia Biotech or Santa Cruz Biotechnology.

\section{Immunofluorescence}

MCF-7 cells were seeded into 4-well slide culture chambers (LabTek). After $24 \mathrm{~h}$, cells were treated with $10 \mu \mathrm{M} \mathrm{CPT}$ for $10 \mathrm{~min}$ or $1 \mathrm{~h}$ or $2 \mathrm{~h}$. Cells were fixed with $4 \%$ paraformaldehyde for $10 \mathrm{~min}$ at room temperature before being permeabilized in $0.5 \%$ Triton X-100. Slide culture chambers were washed with PBS and blocked with PBS containing 2\% BSA, incubated with an antibody specific to FOXO3a or ATM-pS1981 or $\gamma$-H2AX (1:100 to 1:500 dilution), followed by Alexa 546 (red)-conjugated anti-rabbit and Alexa 488 (green)-conjugated antimouse secondary antibodies (Molecular Probes, Eugene, OR). Cells were counterstained with TOPRO-3 (Molecular Probes, Eugene, OR) to show the nuclei. Specific staining was visualized and images were captured with an Olympus IX81 system confocal microscope. Co-localization of two proteins was shown as the merged images. Moreover, quantitative co-localization of FOXO3 with $\gamma-\mathrm{H} 2 \mathrm{AX}$ or ATM-pS1981 was calculated using NIH ImageJ (v. 1.39f) software with the co-localization threshold plug-in (developed by the Wright Cell Imaging Facility, Toronto, Canada). We showed the percentage of image volume as quantitative co-localized pixels between FOXO3 and ATM-pS1981 or $\gamma$-H2AX. To analyze quantitative co-localization, 
we used 50 images randomly captured by confocal microscopy. Each result presented is the mean \pm standard error of the mean (SEM).

\section{Cytoplasmic and nuclear fractionation}

Cells were treated with CPT $(20 \mu \mathrm{M})$ or control (DMSO) for $5 \mathrm{~h}$. Cells were lysed in a lysis buffer (20 mM HEPES, pH7.0, $10 \mathrm{mM} \mathrm{KCl,} 2 \mathrm{mM} \mathrm{MgCl}_{2}, 0.5 \%$ NP-40, $1 \mathrm{mM} \mathrm{Na}_{3} \mathrm{VO}_{4}, 1$ $\mu \mathrm{M}$ phenylmethanesulphonyl-fluoride, and $0.15 \mathrm{units} / \mathrm{ml}$ aprotinin) and homogenized by 30 strokes in a tightly fitting Dounce homogenizer. The homogenate was centrifuged at $1,500 \times g$ for $5 \mathrm{~min}$ to sediment the nuclei. The supernatant was then resedimented at $15,000 \times g$ for $5 \mathrm{~min}$, and then the resulting supernatant formed the cytoplasmic fraction. The nuclei was washed three times and resuspended in PBS containing 0.5\% NP-40 to extract nuclear proteins. The extracted material was sedimented at $15,000 \times g$ for $10 \mathrm{~min}$ and the resulting supernatant was termed the nuclear fraction. Protein concentrations in the cytoplasmic and nuclear fractions were determined with the Bradford protein determination reagent (BioRad Laboratories, Hercules, CA) or BCA Protein Assay Reagent A (PIERCE, Rockford, IL) using BSA as a standard.

\section{Immunocomplex kinase assays}

Flag-FOXO3a protein was immunoprecipitated with an anti-Flag antibody from $1 \mathrm{mg}$ of total cell lysates isolated from $293 \mathrm{~T}$ cells transfected with Flag-FOXO3a expression vector. The endogenous ATM protein complex was immunoprecipitated with an anti-ATM antibody from total lysates $(1 \mathrm{mg})$ isolated from MCF-7 cells, and ATM immunoprecipitates were resuspended in kinase buffer (20 mM HEPES, pH 7.5, $50 \mathrm{mM} \mathrm{NaCl}, 10 \mathrm{mM} \mathrm{MgCl} 2,2.5 \mu \mathrm{M}$ $\beta$-mercaptoethanol, and $200 \mu \mathrm{M}$ ATP). In vitro immunocomplex kinase assays were performed by incubating various amounts of Flag-FOXO3a protein precipitates (FOXO3a-pt) as indicated with equal amounts of endogenous ATM protein complex for $25 \mathrm{~min}$ at $25^{\circ} \mathrm{C}$. As controls, kinase assays were also carried out with or without $50 \mathrm{ng}$ linear double-stranded DNA fragments (mimicking double-strand breaks) (1-kb DNA marker, ISC BioExpress, Kaysville, UT), and in the absence or presence of 20 units of antarctic protein phosphatase (PPase) (New England Biolabs, Ipswich, MA). The final protein products were subjected to $6 \%$ SDSpolyacrylamide gel eletrophoresis, transferred to polyvinylidene difluoride membranes, and analyzed by immunoblotting analysis as described above.

\section{GST pull-down assay}

The various GST-fusion wt and mutant FOXO3a expression vectors were constructed as described in Supplementary Materials and Methods. Briefly, the GST-FO fusion proteins and GST proteins (control) were expressed in Escherichia coli BL21 (Invitrogen, Carlsbad, CA) lysed in a GST lysis buffer (50 mM Tris, $\mathrm{pH} 7.5,150 \mathrm{mM} \mathrm{NaCl}, 1 \%$ Triton X-100, and protease inhibitors), and immobilized onto glutathione-Sepharose beads (Amersham Biosciences). Total lysate $(1 \mathrm{mg})$ from $293 \mathrm{~T}$ cells treated with IR (10 Gy) in binding buffer $(50 \mathrm{mM}$ Tris, $\mathrm{pH} 7.5,100 \mathrm{mM} \mathrm{NaCl}, 10 \mathrm{mM} \mathrm{MgCl} 2,0.5 \%$ Nonidet P-40 and protease inhibitors) was mixed with the GST-FO or GST (control) containing glutathione-Sepharose beads. The protein complex formation on glutathione-Sepharose beads was carried out overnight at $4{ }^{\circ} \mathrm{C}$ with shaking. The beads were washed with binding buffer, and the bound protein complexes were disrupted and proteins were denatured directly by boiling in SDS loading buffer.

\section{Analysis of DNA damage by the comet assay (single-cell gel assay)}

To compare the levels of DNA damage in MCF7-C5, MCF7-FK33, wt FOXO3a(+/+) MEF, and FOXO3a(-/-) MEF cell lines, cells were treated with CPT $(1 \mu \mathrm{M})$ or IR (10Gy) for $1 \mathrm{~h}$ and subjected to a comet assay to detect DNA damage and repair at the level of single cells according to the manufacturer's recommendations (Trevigen, Gaithersburg, MD). Briefly, 
after treatment with CPT or IR, cells were harvested and mixed with low-melting agarose. After lysis, electrophoresis was performed at $1 \mathrm{~V} / \mathrm{cm}$ and $15 \mathrm{~mA}$ for $40 \mathrm{~min}$. Slides were stained with SYBG Green dye for 10min. One hundred randomly selected cells per sample were captured under a Zeiss fluorescent microscope and digital fluorescent images were obtained using the AxioVision software. The relative length and intensity of SYBG Green-stained DNA tails to heads is proportional to the amount of DNA damage present in the individual nuclei and is measured by Olive tail moment using TriTek Comet Score software (TriTek Corp., Sumerduck, VA).

\section{Mammalian two-hybrid assay}

Plasmid clones of pACT-FOXO3a-FL, pACT-FO(301-673), pACT-FO(301-623) mutants, and pBIND-ATM(1802-2571) were generated as described in Supplementary Materials and Methods. 293T cells were plated at $1 \times 10^{5}$ cells per well in 12-well plates. After $24 \mathrm{~h}$, cells were co-transfected with the indicated pACT-FO, pBIND-ATM(1802-2571), and pG5luc vectors at the ratio of 2:1:2 by using GenJet (SignaGen Laboratories, Gaithersburg, MD) according to the manufacturer's instructions. Cells co-transfected with pACT, pBIND, and pG5luc vectors were included as negative controls. After $24 \mathrm{~h}$, cells were lysed in $100 \mu \mathrm{L}$ of Passive Lysis Buffer (Promega Corp. Madison, WI). Firefly and renilla luciferase activities were quantified with the Dual-Luciferase Reporter Assay System (Promega) and a luminometer (Turner BioSystems, Sunnyvale, CA). Relative activity was defined as the ratio of firefly luciferase to renilla luciferase activity.

\section{Statistical analysis}

All data are expressed as means and standard deviations (SD) from at least three determinations. The statistical significance of differences in cell proliferation and tumor growth between two groups was analyzed with two-sided unpaired Student's $t$ tests when the variances were equal, or with Welch's corrected $t$ tests when the variances were unequal, with Graphpad statistical software (San Diego, CA). All statistical tests were two-sided, and P values less than 0.05 were considered statistically significant.

\section{Supplementary Material}

Refer to Web version on PubMed Central for supplementary material.

\section{REFERENCES}

1. Zhou BB, Elledge SJ. The DNA damage response: putting checkpoints in perspective. Nature 2000;408:433-439. [PubMed: 11100718]

2. Shiloh Y. ATM and related protein kinases: safeguarding genome integrity. Nat. Rev. Cancer 2003;3:155-168. [PubMed: 12612651]

3. Kastan MB, Bartek J. Cell-cycle checkpoints and cancer. Nature 2004;432:316-323. [PubMed: 15549093]

4. Savitsky K, et al. A single ataxia telangiectasia gene with a product similar to PI-3 kinase. Science 1995;268:1749-1753. [PubMed: 7792600]

5. Bakkenist CJ, Kastan MB. DNA damage activates ATM through intermolecular autophosphorylation and dimer dissociation. Nature 2003;421:499-506. [PubMed: 12556884]

6. Bakkenist CJ, Kastan MB. Initiating cellular stress responses. Cell 2004;118:9-17. [PubMed: 15242640]

7. Celeste A, et al. Genomic instability in mice lacking histone H2AX. Science 2002;296:922-927. [PubMed: 11934988]

8. Fernandez-Capetillo O, Celeste A, Nussenzweig A. Focusing on foci: H2AX and the recruitment of DNA-damage response factors. Cell Cycle 2003;2:426-427. [PubMed: 12963833] 
9. Lee JH, Paull TT. ATM activation by DNA double-strand breaks through the Mre11-Rad50-Nbs1 complex. Science 2005;308:551-554. [PubMed: 15790808]

10. Burgering BM, Kops GJ. Cell cycle and death control: long live Forkheads. Trends Biochem. Sci 2002;27:352-360. [PubMed: 12114024]

11. Tran H, Brunet A, Griffith EC, Greenberg ME. The Many Forks in FOXO's Road. Sci. STKE 2003;2003:re5. [PubMed: 12621150]

12. Brunet A, et al. Akt promotes cell survival by phosphorylating and inhibiting a Forkhead transcription factor. Cell 1999;96:857-868. [PubMed: 10102273]

13. Accili D, Arden KC. FoxOs at the crossroads of cellular metabolism, differentition, and transformation. Cell 2004;117:421-426. [PubMed: 15137936]

14. Hu MC-T, et al. IкB kinase promotes tumourigenesis through inhibition of Forkhead FOXO3a. Cell 2004;117:225-237. [PubMed: 15084260]

15. Kops GJ, Dansen TB, Polderman PE, et al. Forkhead transcription factor FOXO3a protects quiescent cells from oxidative stress. Nature 2002;419:316-321. [PubMed: 12239572]

16. Furukawa-Hibi Y, Yoshida-Araki K, Ohta T, Ikeda K, Motoyama N. FOXO forkhead transcription factors induce G(2)-M checkpoint in response to oxidative stress. J. Biol. Chem 2002;277:2672926732. [PubMed: 12048180]

17. Essers MA, et al. Functional interaction between b-catenin and FOXO in oxidative stress signaling. Science 2005;308:1181-1184. [PubMed: 15905404]

18. Goldberg M, et al. MDC1 is required for the intra-S-phase DNA damage checkpoint. Nature 2003;421:952-956. [PubMed: 12607003]

19. d'Adda di Fagagna F, et al. A DNA damage checkpoint response in telomere-initiated senescence. Nature 2003;426:194-198. [PubMed: 14608368]

20. Wu ZH, Shi Y, Tibbetts RS, Miyamoto S. Molecular linkage between the kinase ATM and NF-кB signaling in response to genotoxic stimuli. Science 2006;311:1141-1146. [PubMed: 16497931]

21. Frescas D, Valenti L, Accili D. Nuclear trapping of the forkhead transcription factor FoxO1 via Sirtdependent deacetylation promotes expression of glucogenetic genes. J. Biol. Chem 2005;280:20589_ 20595. [PubMed: 15788402]

22. Niida H, Nakanishi M. DNA damage checkpoints in mammals. Mutagenesis 2006;21:3-9. [PubMed: 16314342]

23. Tran $\mathrm{H}$, et al. DNA repair pathway stimulated by the forkhead transcription factor FOXO3a through the Gadd45 protein. Science 2002;296:530-534. [PubMed: 11964479]

24. Matijasevic Z, Precopio ML, Snyder JE, Ludlum DB. Repair of sulfur mustard induced DNA damage in mammalian cells measured by a host cell reactivation assay. Carcinogenesis 2001;22:661-664. [PubMed: 11285203]

25. Bosotti R, Isacchi A, Sonnhammer EL. FAT: a novel domain in PIK-related kinases. Trends Biochem. Sci 2000;25:225-227. [PubMed: 10782091]

26. Fernandez-Capetillo O, et al. DNA damage-induced G2-M checkpoint activation by histone H2AX and 53BP1. Nat. Cell Biol 2002;4:993-997. [PubMed: 12447390]

27. Osborn AJ, Elledge SJ, Zou L. Checking on the fork: the DNA-replication stress-response pathway. Trends Cell Biol 2002;12:509-516. [PubMed: 12446112]

28. Bartkova J, et al. DNA damage response as a candidate anti-cancer barrier in early human tumourigenesis. Nature 2005;434:864-870. [PubMed: 15829956]

\section{ACKNOWLEDGEMENTS}

We thank M.B. Kastan, R. Depinho, N. Motoyama, S. Miyamoto, A.J. Fornace Jr., and K.K. Khanna for generously providing reagents. This work was supported in part by R01 grant CA113859 (to M.C.T.H.) from the National Cancer Institute (NCI), National Institutes of Health; grants BCTR0504415 from the Susan G. Komen Breast Cancer Foundation, BC045295 from the U.S. Department of Defense Breast Cancer Research Program, and a grant from the Texas Advanced Research Program (to M.C.T.H.); and Cancer Center Support Grant CA16772 from the NCI. The sponsors had no role in the design, conduct, or reporting of the study. 

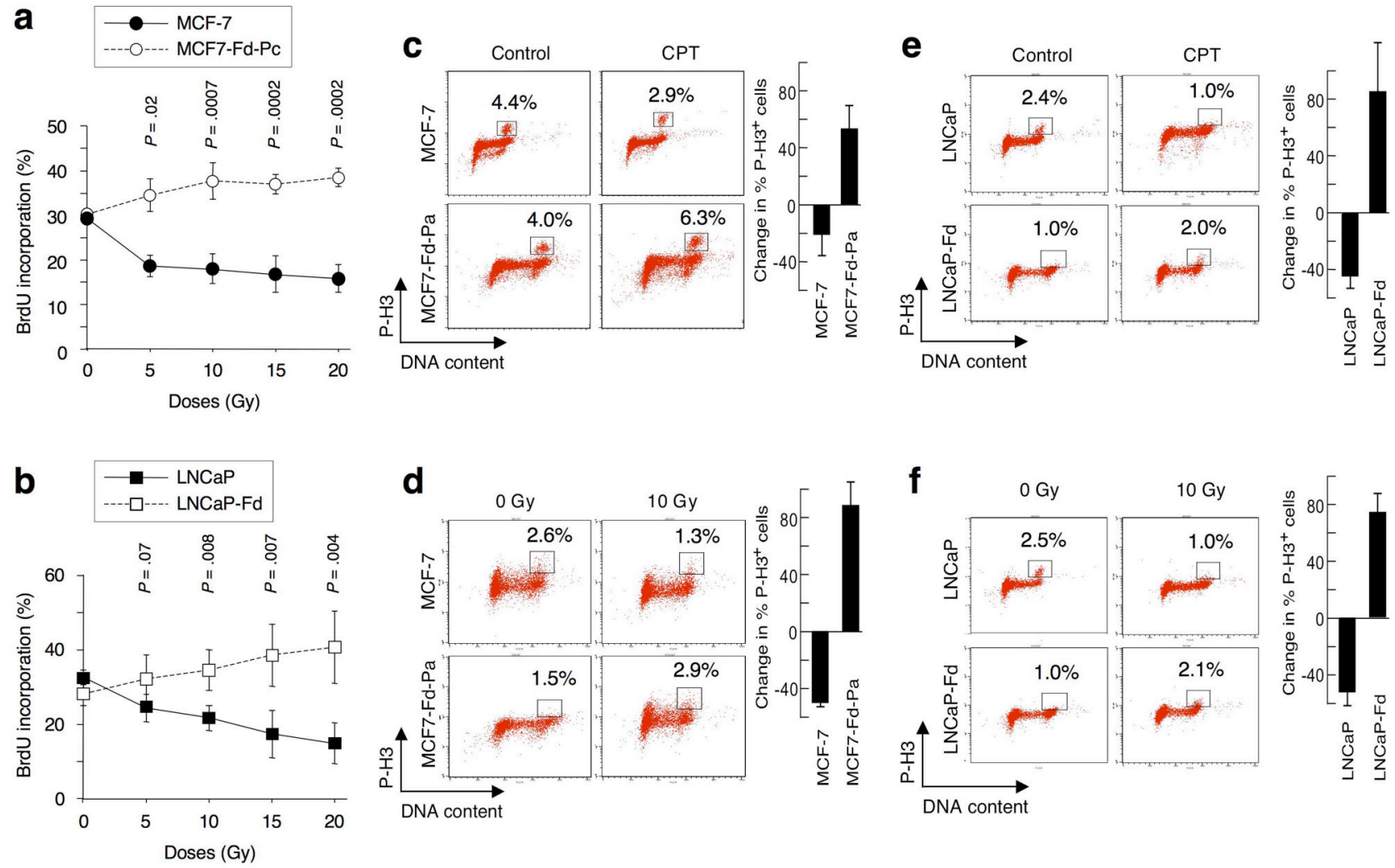

Figure 1.

Silencing FOXO3a results in defective intra-S-phase and $\mathrm{G}_{2} / \mathrm{M}$ phase cell-cycle checkpoints. (a) Analysis of the IR-induced intra-S-phase checkpoint. MCF-7 and MCF7-Fd-Pc (FOXO3adownregulation pooled clones) cells were untreated or treated with various doses of IR, incubated for $1 \mathrm{~h}$ at $37^{\circ} \mathrm{C}$, then labeled with $\mathrm{BrdU}$ for $30 \mathrm{~min}$ at $37^{\circ} \mathrm{C}$ before fixation. DNA synthesis in replication was determined by flow cytometry. (b) LNCaP and LNCaP-Fd (FOXO3a-downregulation cell line) were untreated or treated with various doses of IR as indicated. DNA synthesis was measured as described above. (c) Analysis of the $\mathrm{G}_{2} / \mathrm{M}$ DNA damage checkpoint. MCF-7 and MCF7-Fd-Pa were treated with camptothecin (CPT) $(1 \mu \mathrm{M})$ or control (DMSO) for $1 \mathrm{~h}$. Cells in mitosis were assessed by staining with an antibody against phosphorylated histone-H3 at Serine-10 (P-H3) or an isotype IgG (negative control), followed by an Alexa 488-conjugated secondary antibody and propidium iodide (PI). The percentage of P-H3-positive cells was determined by flow cytometry. (d) Similarly, MCF-7 and MCF7-Fd$\mathrm{Pa}$ were irradiated with $10 \mathrm{~Gy}$ or untreated $(0 \mathrm{~Gy})$ for $1 \mathrm{~h}$, and the percentage of $\mathrm{P}-\mathrm{H} 3$-positive cells was measured. (e) LNCaP and LNCaP-Fd were treated with CPT ( $1 \mu \mathrm{M})$ or control for $1 \mathrm{~h}$, and the percentage of P-H3-positive cells was determined. (f) LNCaP and LNCaP-Fd were irradiated with 10Gy or untreated for $1 \mathrm{~h}$, and the percentage of P-H3-positive cells was measured as described above. The changes in percentage of P-H3-positive cells between treatment (CPT or IR) and control shown in histograms represent the averages of three independent experiments. The $P$ values between MCF-7 versus MCF7-Fd-Pc and between LNCaP versus LNCaP-Fd are indicated. All statistical tests were two-sided, and $P$ values less than 0.05 were considered statistically significant. 
a

C
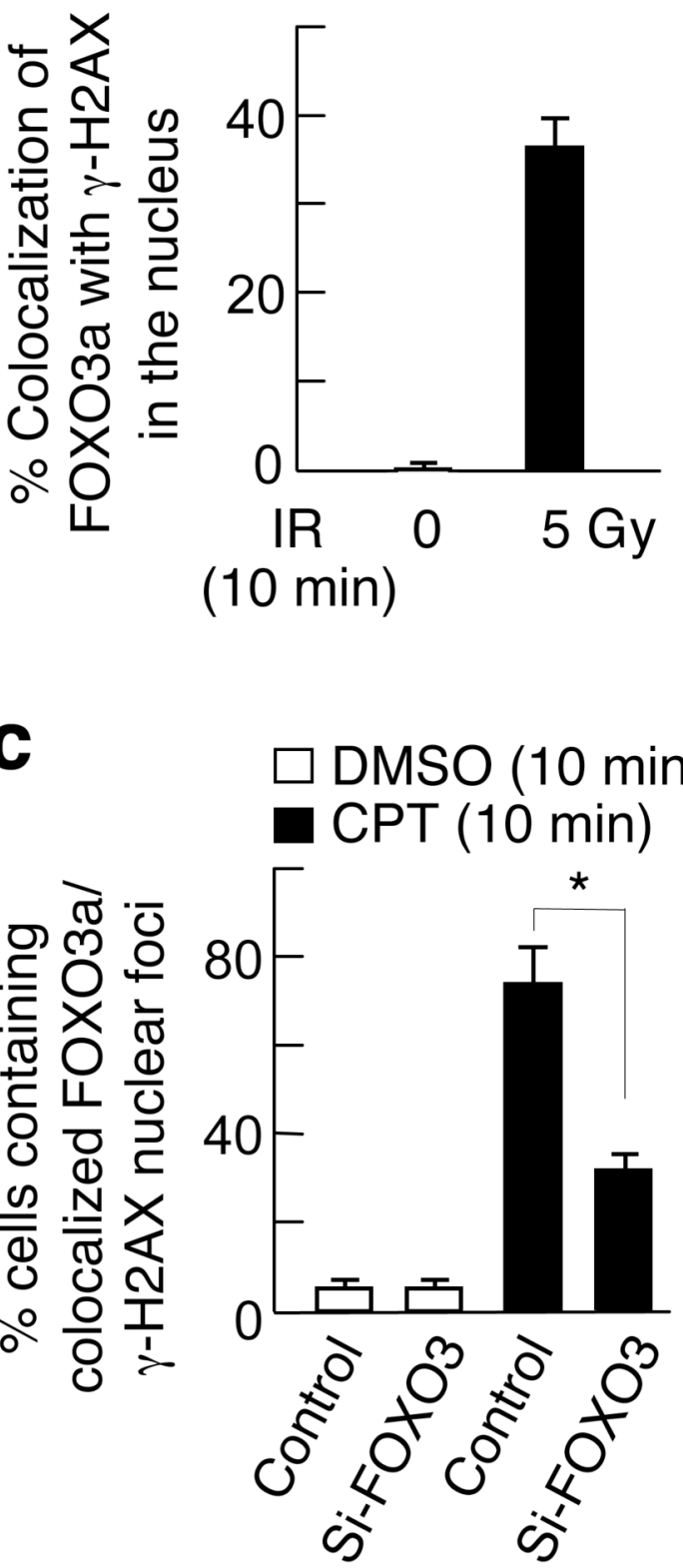

b
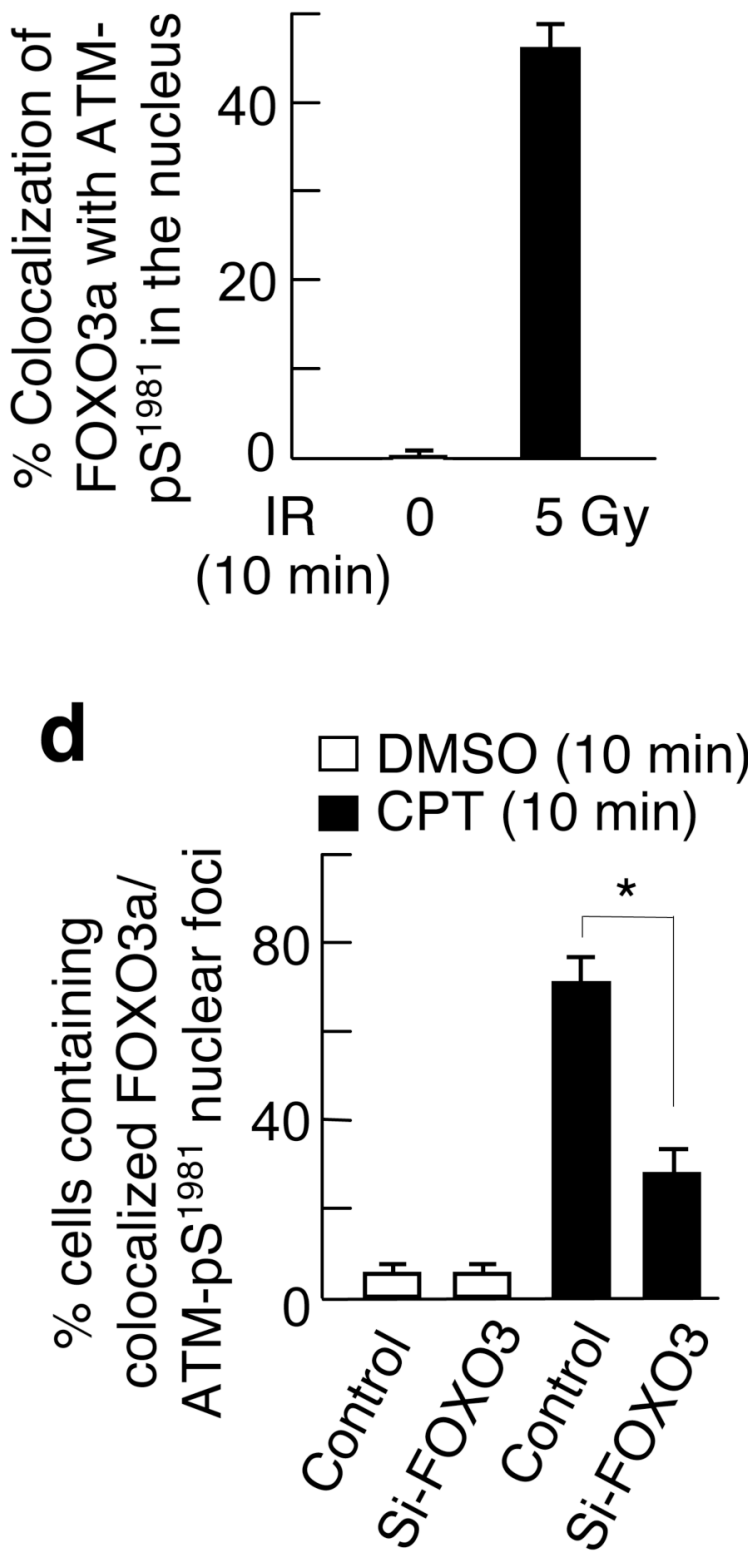

Figure 2.

FOXO3a may be co-localized with $\gamma$-H2AX and ATM-pS1981 to form nuclear foci in cells treated with ionizing radiation (IR). MCF-7 cells were treated with IR ( 5 Gy) or untreated ( 0 Gy) for 10min, fixed, and the subcellular localizations and co-localization of endogenous FOXO3a and (a) $\gamma$-H2AX or (b) ATM-pS1981 were detected using antibodies specifically against FOXO3a and $\gamma$-H2AX and followed by an Alexa Fluor 546(red)- or Alexa Fluor 488 (green)-conjugated secondary antibody, respectively, and fluorescence microscopy. The percentage of quantitative co-localization of FOXO3a with $\gamma$-H2AX (a) or ATM-pS1981 (b) was calculated using NIH ImageJ (v. 1.39f) software with the co-localization program. An average of 50 merged nuclear foci on three independent slides was determined. The results 
presented are the mean \pm SEM. (c, d) MCF-7 cells were transfected with siRNA targeting FOXO3a (si-FOXO3) or control, treated with CPT or DMSO for $10 \mathrm{~min}$, and then the subcellular localizations of $\gamma$-H2AX and ATM-pS1981 were detected using specific antibodies. The averages of $>300$ cells with co-localized FOXO3a and $\gamma$-H2AX (c) or ATM-pS1981 (d) nuclear foci on three independent slides were determined. *, $P \&<0.001$ between control and si-FOXO3 after DNA damage for 10min. Representative pictures and their higher power views of $\mathbf{c}$ and $\mathbf{d}$ are shown in Supplementary-Fig. S3e-h. 

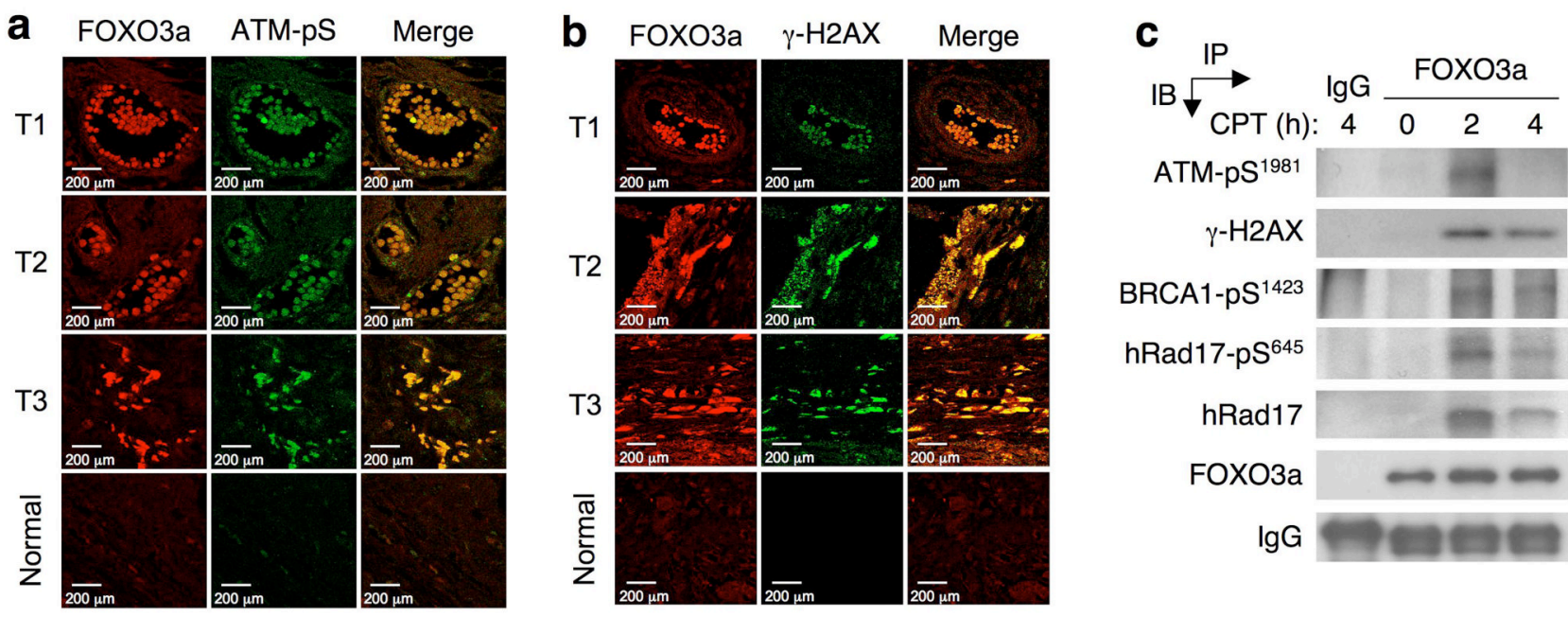

d

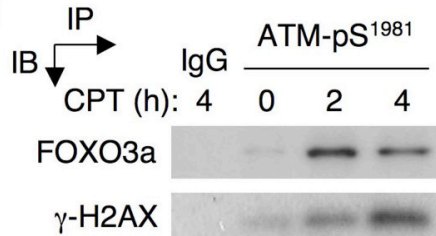

BRCA1-pS ${ }^{1423}$

hRad17-pS 645

hRad17

$\lg G$ e

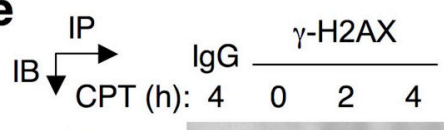

FOXO3a

$\gamma-\mathrm{H} 2 \mathrm{AX}$

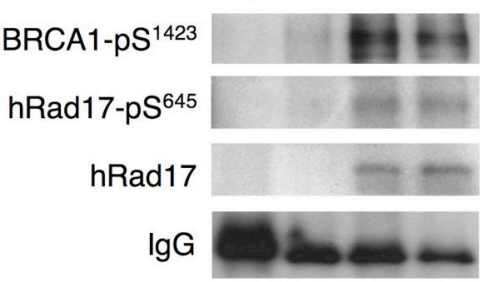

f $\begin{array}{lllllll}\text { FOXO3a-pt } & 0 & 1 & 5 & 10 & 10 & (\mu \mathrm{l})\end{array}$ $\stackrel{\mathrm{IP}}{\longrightarrow} \quad$ ATM ${ }^{\operatorname{lgG}} \mathrm{kDa}$ ATM-pS ${ }^{1981} \quad 370$
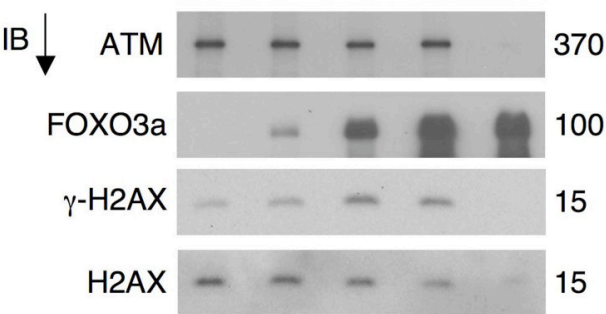

Figure 3.

FOXO3a is co-localized with ATM-pSer1981 or $\gamma$-H2AX in primary tumour tissues in vivo, associated with these phospho-proteins in the nucleus in cells treated with CPT, and involved in ATM autophosphorylation of Ser-1981. (a, b) Nuclear FOXO3a is co-localized with ATMpS1981 (a) or $\gamma$-H2AX (b) in human breast tumour tissues. Human primary breast tumour tissues (T) and normal tissues were stained with antibodies against FOXO3a and ATM-pS1981 or $\gamma-\mathrm{H} 2 \mathrm{AX}$ as described. Co-localization of nuclear FOXO3a with ATM-pS1981 or $\gamma-\mathrm{H} 2 \mathrm{AX}$ was shown as the merged images. Scale bars indicate $200 \mu \mathrm{m}$. (c) Nuclear extracts of MCF-7 cells treated with CPT $(20 \mu \mathrm{M})$ for $2 \mathrm{~h}$ or $4 \mathrm{~h}$ or untreated $(0 \mathrm{~h})$ were subjected to immunoprecipitation (IP) with an anti-FOXO3a antibody followed by immunoblotting (IB) analysis with an antibody against ATM-pS1981 or $\gamma$-H2AX or BRCA1-pS1423 or hRad17pS645 or hRad17 or FOXO3a (IP positive control) or an isotype IgG (negative control). (d, e) Similarly, nuclear extracts of MCF-7 cells treated with CPT were subjected to IP with an antibody against ATM-pS1981 (d) or $\gamma$-H2AX (e) followed by IB with an antibody against FOXO3a or the indicated proteins or an IgG (control). (f) FOXO3a promotes ATM autophosphorylation of S1981. In vitro immunocomplex kinase assays were performed by incubating various amount of FOXO3a protein (FOXO3a-pt), which were immunoprecipitated using an anti-Flag antibody from 293T lysates transfected with Flag-FOXO3a expression vector, with equal amount of endogenous ATM protein complex obtained from MCF-7 cells by IP. The levels of phosphorylated ATM (ATM-pS1981), ATM, $\gamma$-H2AX, H2AX, and FOXO3a were determined by IB analysis with specific antibodies as highlighted. All IP and 
IB experiments were repeated at least twice. The uncropped images of $\mathbf{c}, \mathbf{d}, \mathbf{e}$ and $\mathbf{f}$ are shown in Supplementary-Fig. S6. 

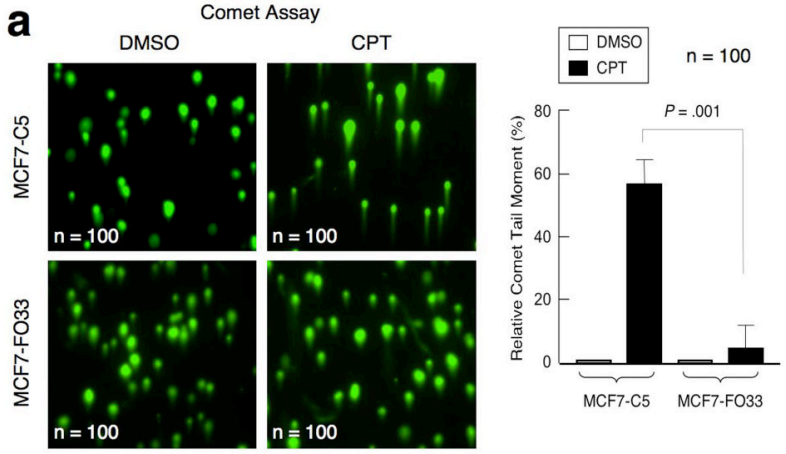

C
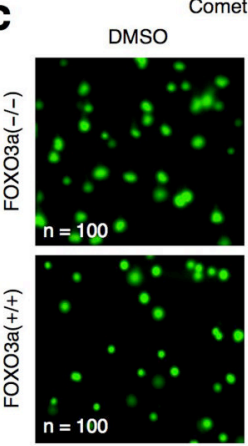

Comet Assay
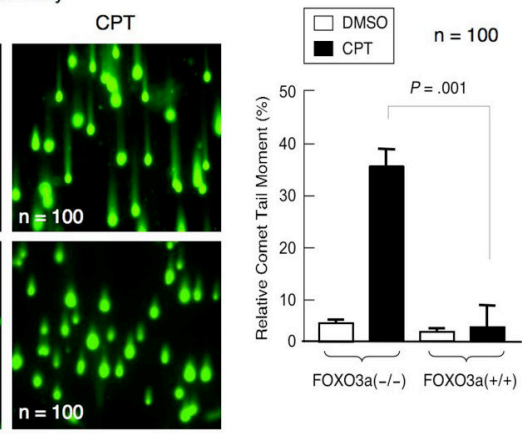
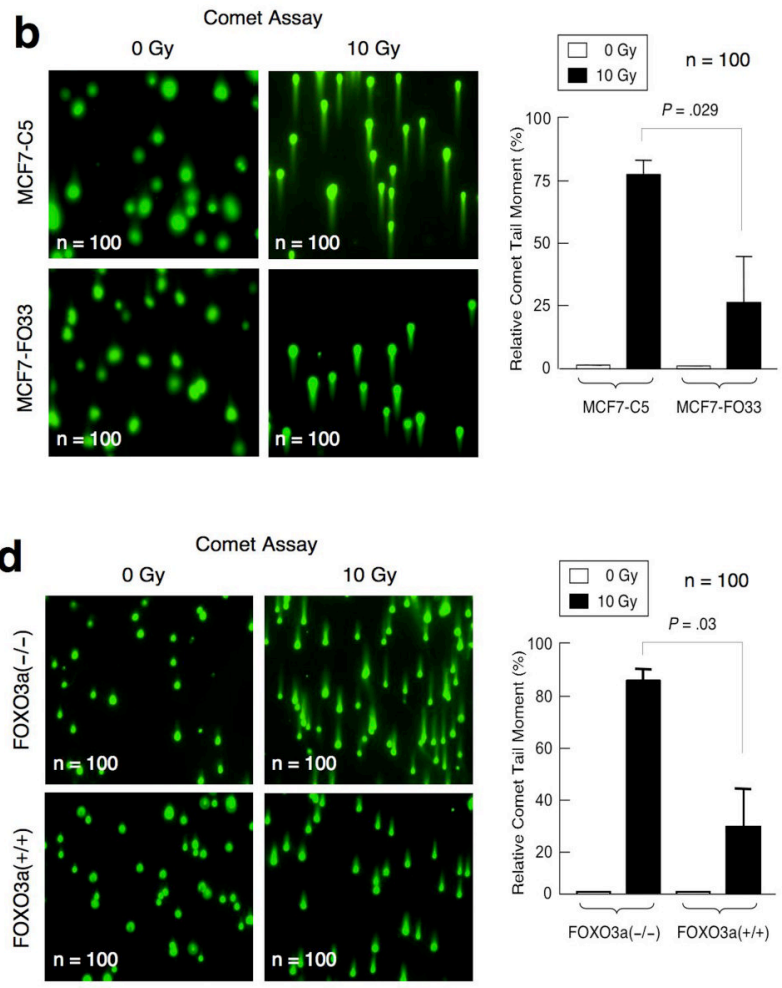

Figure 4.

FOXO3a promotes the repair of damaged DNA. (a) MCF7-C5 and MCF7-FO33 cells were treated with CPT $(1 \mu \mathrm{M})$ or control (DMSO) for $1 \mathrm{~h}$, and the comet assay (single-cell electrophoresis) was done immediately. The relative length and intensity of SYBG Greenstained DNA tails to heads (shown as \% of the relative comet tail moment) is proportional to the amount of DNA damage present in the individual nuclei $(\mathrm{n}=100)$. (b) MCF7-C5 and MCF7FO33 were treated with IR (10 Gy), and single-cell electrophoresis was done $1 \mathrm{~h}$ after IR $(\mathrm{n}=100)$. (c) Wild-type FOXO3a $(+/+)$ and FOXO3a(-/-) MEFs were treated with CPT $(1 \mu \mathrm{M})$ or control (DMSO) for $1 \mathrm{~h}$, single-cell electrophoresis was performed, and the relative comet tail moment was determined $(\mathrm{n}=100)$. (d) Wild-type FOXO3a $(+/+)$ and FOXO3a(-/-) MEFs were treated with IR (10 Gy), and single-cell electrophoresis was carried out $1 \mathrm{~h}$ after IR, and the relative comet tail moment was measured. The changes in percentage of the relative comet tail moment between treatment (CPT or IR) and control shown in all histograms represent the averages of three independent experiments $(\mathrm{n}=100)$. The $P$ values between MCF7-C5 versus MCF7-FO33 and between FOXO3a $(+/+)$ versus FOXO3a(-/-)MEFs are highlighted. 


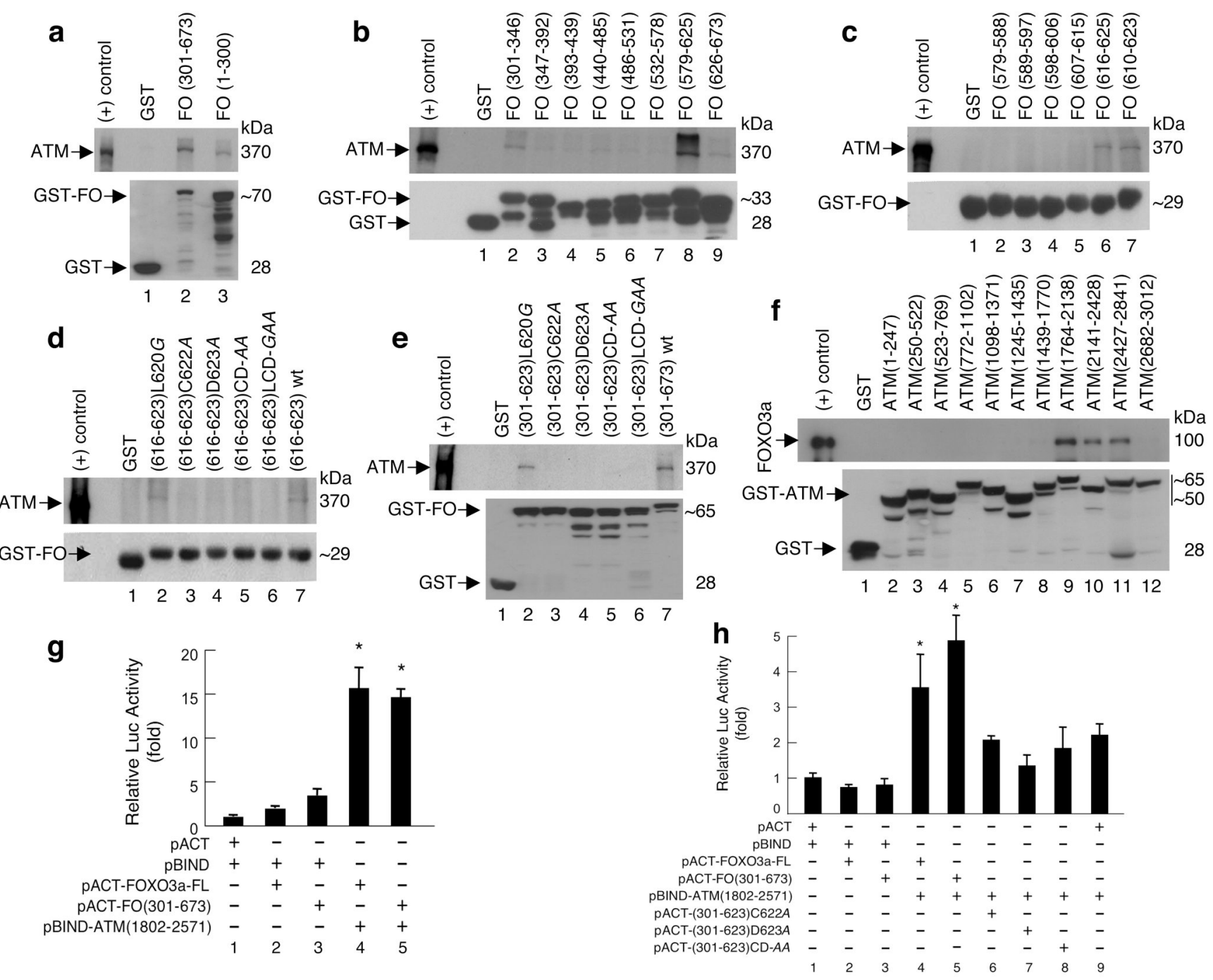

Figure 5.

The carboxy-terminal domain of FOXO3a binds to the FAT domain of ATM in vitro and in vivo. (a) GST-pull down in vitro assays. Lysates from 293T cells treated with IR (10 Gy) were incubated with the GST-FOXO3a (GST-FO) fusion proteins as indicated and GST alone (negative control), and analyzed by immunoblotting with antibodies against ATM and GST. (b) Lysates were incubated with the eight GST-FO sequential fragments spanning the region of FOXO3a(301-673) as highlighted and GST, and analyzed as described above. (c) Lysates were incubated with the six GST-FO sequential fragments spanning a specific regain (579625) that showed the strongest signal in binding to ATM in b. (d) Lysates were incubated with the GST-fusion proteins containing the short (8-a.a.) motif of wt FO(616-623) and five mutant FO(616-623) fragments as indicated, and GST, and analyzed. (e) Lysates were incubated with the GST-fusion proteins containing the C-terminal domain (323-a.a.) of five mutant FO(301623) fragments as indicated, wt FO(301-673) (positive control) and GST, and analyzed. (f) Lysates from 293T transfected with FOXO3a vector were incubated with GST-ATM proteins as indicated, and GST, and immunoblotting with antibodies against FOXO3a and GST. (g) Mammalian two-hybrid analysis. The pACT-FOXO3a-FL (full-length), pACT-FO(301-673), pBIND-ATM(1802-2571), and empty vectors (pACT and pBIND) were transfected into 293T along with the GAL4-firefly-luciferase (Luc) reporter plasmid, pG5luc, as indicated. Identical 
amounts of DNA were transfected into each well, and lysates were harvested 24h post transfection. Firefly-Luc activity was normalized with renilla-Luc activity, and quantified relative to empty pACT and pBIND co-transfected cells. The relative Luc activities presented show the average of three independent experiments with error bars indicating standard deviations (SD). $* P \&<0.0007$ between the groups of pBIND (control) binding to pACT-FO [FOXO3a-FL or (301-673)] versus the groups of pBIND-ATM(1802-2571) binding to pACTFO. (h) The indicated vectors were transfected into $293 \mathrm{~T}$ along with pG5luc. The relative Luc activities were determined. $* P \&<0.007$ between the groups of pBIND (control) binding to pACT-FO [FOXO3a-FL or (301-673)] versus the groups of pBIND-ATM(1802-2571) binding to pACT-FO. 